\title{
Revealing a Pauli Crystal
}

\author{
A novel, high-resolution fluorescence imaging technique reveals a \\ pattern, known as a Pauli crystal, that can emerge in a cloud of trapped, \\ noninteracting fermions.
}

\author{
By Christie Chiu
}

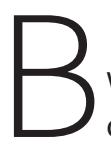

ring two particles together and, in general, they will interact. For example, two electrons will repel each other through electrostatic forces, or two atoms may form a molecule through electrostatic and van der Waals forces. Noninteracting particles, however, can also affect another's behavior in a way that depends on the spin of both particles. In particular, fermionic particles, which have half-integer spin, obey the Pauli exclusion principle, which states that two fermions can never occupy the same quantum state. Two electrons in an atom, for instance, can never occupy the same quantum state. As a result, noninteracting particles can form self-organized structures. However, these structures, called Pauli crystals, have not been previously observed. Now using ultracold atoms, Marvin Holten from the University of Heidelberg, Germany, and colleagues have experimentally

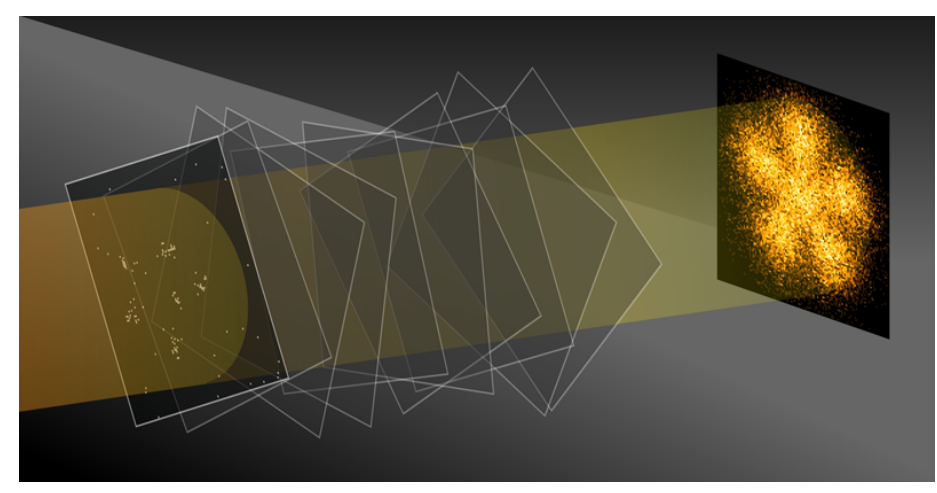

Figure 1: As a consequence of the Pauli exclusion principle, a few fermions trapped by lasers self-organize into a periodic structure called a Pauli crystal. By combining single-particle snapshots of the system, Marvin Holton and colleagues measure the multiparticle correlations that reveal the formation of a Pauli crystal.

Credit: APS/Alan Stonebraker realized and imaged a Pauli crystal [1]. The team's approach holds the promise of microscopically studying many-particle correlations in two-dimensional Fermi gases, which could help in explaining phenomena such as unconventional superconductivity.

Although the behavior of one or pairs quantum particles is well-understood, that of larger, many-body quantum systems is an active area of research. In the past few decades, ultracold atoms have emerged as a powerful experimental platform for studying these systems. Today, scientists can cool ensembles of atoms to temperatures so low that they are well within the quantum regime in which their wave-like properties become apparent. We can place the cold atoms in a highly controlled potential landscape, or environment, created using laser light. And we can probe these systems by imaging the atomic ensemble and analyzing the resulting images. We can even image individual atoms and determine their mutual correlations [2-4]. However, these experiments hold single atoms in arrays of discrete traps, or lattice potentials, which are explicitly designed for studies of quantum many-body physics in crystalline solids. In contrast, the demonstration of Pauli crystals would require observation of self-organization in a single trap.

In a semiclassical picture, at zero temperature, particles are drawn to a trap's minimum potential energy. Yet in a trap that holds multiple fermions, not every particle can be at the minimum energy. As a result, the particles organize into a geometric, ordered structure relative to each other-picture a flower- or wheel-like arrangement, where the number of petals or spokes changes depending on how many atoms are contained in the trap. The Pauli crystal mirrors the symmetry of the trap. A disk-shaped trap, for example, has continuous 
rotational symmetry and should give rise to a many-fermion wave function that does not change under rotation. The term "crystal" derives from a loose analogy with the geometric regularity of real crystals, but Pauli crystals exhibit neither the translational symmetry nor the long-range order that define a real crystal. The relative positions and momenta of the particles in the Pauli crystal are described by continuous variables, in contrast to the discrete variables that define the specific sites of a crystal lattice.

The researchers create a Pauli crystal by using lasers to trap clouds of three or six lithium- 6 atoms, which are electrically neutral fermions. All the atoms are prepared in the same internal quantum state and lie at the bottom of a two-dimensional, rotationally symmetric harmonic potential. With a sufficiently tight trap and at sufficiently low temperatures, Pauli exclusion becomes significant, and the atoms self-organize into geometric patterns. At this point, the atoms are only a micrometer or two apart from each other and can't be directly imaged. The team instead extracts their distribution through a time-of-flight expansion: by relaxing the radial confinement, atoms move outward based on their momenta. At the same time, the weak axial confinement is maintained, which keeps the atoms in the 2D plane.

Holten and colleagues' snapshots reveal the formation of the Pauli crystal, in which observed atom locations depend on their momenta while in the trap. To create these snapshots, the researchers use a fluorescence imaging technique developed by their group for single-atom imaging of unconfined ultracold atoms [5]. By collecting a mere 20 photons per atom, they avoid heating the atoms significantly, thereby removing the need for additional laser cooling. As a result, the scheme allows them to probe the correlations of the momenta of many fermions while maintaining single-atom resolution.

Because each individual image provides a single sample of the many-body wave function, it measures the momentum of every atom but not the atoms' geometric order. To reveal the rotational symmetry of the many-body wave function, thousands of these images would have to be averaged, and each one re-centered about the system's center of mass. However, each snapshot captures a different angular orientation of the system, so the overall geometric order would be lost in the averaging. To overcome this problem and confirm that the structures are indeed Pauli crystals, the researchers rotate each image prior to averaging [6] (Fig. 1). Finally, by shaking the trap in a controlled manner to add energy to their system, the researchers reduce the order and melt their Pauli crystal. By comparing images of this melting to numerical simulations, the researchers conclude that the structural contrast of their Pauli crystals could be improved by reaching even lower temperatures.

The experiment suggests that their technique could be applied to any ensemble of atoms in a $2 \mathrm{D}$ trap, including interacting atoms. With the addition of spin sensitivity, the new imaging capability will enable few-body studies of interacting fermionic spins across a wide range of interaction strengths and temperatures.

A bigger challenge will be to scale up the experiment to larger ensembles of particles and investigate how the physics changes as the system's size increases. But if successful, doing so will provide an unprecedented view into 2D Fermi gases. The measurement of local particle densities and of multiparticle correlation patterns may allow us to connect the microscopic behavior of many-body systems to emergent macroscopic phenomena. By better understanding how fermions pair up, how paired fermions produce a superfluid, or how a single spin impurity is affected by its surroundings, we may finally decipher intriguing systems such as strange metals and unconventional superconductors.

Christie Chiu: Department of Electrical Engineering and the Princeton Center for Complex Materials, Princeton University, Princeton, NJ, USA

\section{REFERENCES}

1. M. Holten et al., "Observation of Pauli crystals," Phys. Rev. Lett. 126, 020401 (2021).

2. M. F. Parsons et al., "Site-resolved measurement of the spin-correlation function in the Fermi-Hubbard model," Science 353, 1253 (2016).

3. M. Boll et al., "Spin- and density-resolved microscopy of antiferromagnetic correlations in Fermi-Hubbard chains," Science 353, 1257 (2016).

4. L. W. Cheuk et al., "Observation of spatial charge and spin correlations in the 2D Fermi-Hubbard model," Science 353, 1260 (2016). 
5. A. Bergschneider et al., "Spin-resolved single-atom imaging of ${ }^{6} \mathrm{Li}$ in free space," Phys. Rev. A 97, 063613 (2018).
6. M. Gajda et al., "Single-shot imaging of trapped Fermi gas," Europhys. Lett. 115, 20012 (2016). 